\title{
A Population of In Silico Models to Face the Variability of Human Induced Pluripotent Stem Cell-derived Cardiomyocytes: the hERG Block Case Study
}

\author{
Michelangelo Paci ${ }^{1}$, Elisa Passini ${ }^{2}$, Stefano Severi ${ }^{3}$, Jari Hyttinen ${ }^{1}$, Blanca Rodriguez ${ }^{2}$ \\ ${ }^{1}$ ELT, Tampere University of Technology, BioMediTech, Tampere, Finland \\ ${ }^{2} \mathrm{CS}$, University of Oxford, Oxford, United Kingdom \\ ${ }^{3}$ DEI, University of Bologna, Cesena, Italy
}

\begin{abstract}
Human induced pluripotent stem cell-derived cardiomyocytes (hiPSC-CMs) are characterized by an extreme variability, which cannot be reproduced by a single in silico model. Here we present a population of hiPSC-CM models, calibrated using six different experimental datasets. By sampling the maximum conductances of 11 ionic currents, 10000 parameter sets were obtained. The experimental data-based calibration selected 1355 in silico models to be included in the final population. Such population reproduces the experimental data variability and it is used to assess the different responses to a 90\% $I_{K r}$ block. Three different profiles emerged: models still normally beating (562), action potentials with EADs (336) and repolarization failures (457). The models still beating after $800 \mathrm{~s}$ since $I_{K r}$ block showed a mean $\triangle A P D_{90}$ of $723 \pm 12$ ms. We observed significant differences among these three classes in the maximum conductances of $I_{C a L}, I_{K r}, I_{K s}, I_{K 1}, I_{N a C a}$ and $I_{N a K}$, supporting the idea that hiPSC-CM belonging to the same control population can however show dramatically different responses to an external perturbation, due to the physiological variability. This has to be taken in proper consideration in the perspective of using hiPSC-CMs for safety pharmacology assays.
\end{abstract}

\section{Introduction}

During the last decade, the potential of human induced pluripotent stem cell-derived cardiomyocytes (hiPSCCMs) has been remarked for many different applications, ranging from regenerative medicine, to personalized medicine, to drug tests. The potential of hiPSC-CMs for drug testing was acknowledged also in the new Comprehensive In vitro Proarrhythmia Assay (CiPA) paradigm for drug safety testing. In fact, CiPA aims to combine in vitro (hiPSC-CMs) and in silico (computational models of cardiac cells) technologies to overcome the limits of the current safety pharmacology paradigm (i.e. the focus on hERG channel block in vitro and the QT prolongation in vivo) [1]. However, the use of hiPSC-CMs poses new challenges, e.g. differences compared to adult cardiac [2] cells and the extreme variability of the experimental data. hiPSC-CMs variability represent a big challenge, as shown by plethora of in vitro datasets published during the last five years. Recently, a hiPSC-CM in silico model was proposed (Paci2015) [2,3], but due to its intrinsic development, it cannot capture the hiPSC-CM variability since i) it reproduces the average behaviour of an in vitro hiPSCCMs population and ii) such population refers to a single dataset [4]. The aim of this paper is tackling the hiPSCCMs variability issue in silico, using the new "population of models" (POM) paradigm [5]. In detail, we aim to overcome the limits of the Paci2015 model and capture the variability observed in literature, with particular focus on the effects of a strong block of $\mathrm{I}_{\mathrm{Kr}}$, which has been reported to induce Early After Depolarizations (EADs), in some cells but not in others. E.g. in [4] only three out of five cells showed EADs, but the Paci2015 model did not simulate such phenomenon.

\section{Methods}

The POM paradigm consists in two separate steps: i) the generation of a first population of in silico models, by sampling the parameters of interest and ii) calibrating the first population using the available experimental data, thus discarding those model which are not in agreement with the experimental evidences. We followed these two steps to obtain an in silico hiPSC-CM population, which is then used to test a strong blockade of $\mathrm{I}_{\mathrm{Kr}}$.

\subsection{Parameter sampling and model generation}

The first population was generated using the Paci2015 model and sampling the maximum conductances of 11 currents: fast and late $\mathrm{Na}^{+}\left(\mathrm{I}_{\mathrm{Na}}\right.$ and $\left.\mathrm{I}_{\mathrm{NaL}}\right)$, L-type $\mathrm{Ca}^{2+}$ ( $\left.\mathrm{I}_{\mathrm{CaL}}\right)$, funny $\left(\mathrm{I}_{\mathrm{f}}\right.$ ), transient outward $\mathrm{K}^{+}\left(\mathrm{I}_{\mathrm{to}}\right)$, rapid and 
slow delayed rectifier $\mathrm{K}^{+}\left(\mathrm{I}_{\mathrm{Kr}}\right.$ and $\mathrm{I}_{\mathrm{Ks}}$ ), inward rectifier $\mathrm{K}^{+}$ ( $\left.\mathrm{I}_{\mathrm{K} 1}\right), \mathrm{Na}^{+}-\mathrm{Ca}^{2+}$ exchanger $\left(\mathrm{I}_{\mathrm{NaCa}}\right), \mathrm{Na}^{+}-\mathrm{K}^{+}$pump ( $\mathrm{I}_{\mathrm{NaK}}$ ) and sarcolemmal $\mathrm{Ca}^{2+}$ pump $\left(\mathrm{I}_{\mathrm{pCa}}\right)$. The only change in the Paci2015 model is a speeded up $\mathrm{I}_{\mathrm{CaL}}$ recovery from inactivation (time constants $\mathrm{x}$ 0.4), to stress the arrhythmogenic phenomena. The sampling technique is the Latin Hypercube Sampling (LHS) [5], which allows an even sampling of the parameter spaces. LHS produced 10000 sets of 11 coefficients each, ranging in $[0.5,2]$ which were used to modulate the aforementioned 11 currents, thus generating a first population of 10000 hiPSC-CM models. Simulations were run for $800 \mathrm{~s}$ to drive the models to the steady state.

\subsection{Calibration of the first population}

To calibrate the hiPSC-CM population, we used six datasets of spontaneous action potentials (APs) available in literature, namely Ma2011 [4], Moretti2010 [6], Ma2013 [7], Fatima2013 [8], Lahti2012 [9] and Kujala2012 [10], where biomarkers such as AP duration (APD), maximum diastolic potential (MDP) and rate of spontaneous beating (RATE) where reported in terms of mean $(\mu)$ and standard error (SEM). For each biomarker we consider the biomarker space as $\mu \pm 2$ standard deviations $(\sigma)$, hypothesizing a uniform distribution. Due to the variability of the datasets, we joined them together in a conservative way, i.e. considering as lower and upper bounds for each biomarker the smallest and the biggest among the six datasets, respectively (Table 1). Not meaningful bounds (e.g. negative RATE or APD) were set to 0 . In order for a model to be included into the final population, every biomarker must be included in the aforementioned biomarker spaces. Since we focused on spontaneously beating in silico models, we introduced two more conditions than in [5] to accept a model into the final population: i) the model must generate spontaneous APs and ii) the intracellular ionic concentrations must be $5 \leq\left[\mathrm{Na}^{+}\right]_{\mathrm{i}} \leq 15 \mathrm{mM}$ and $0 \leq\left[\mathrm{Ca}^{2+}\right]_{\mathrm{SR}} \leq 5 \mathrm{mM}$.

\subsection{IKr block}

To assess the behaviour of the in silico hiPSC-CM population we tested an extreme $\mathrm{I}_{\mathrm{Kr}}$ block (90\%) for $800 \mathrm{~s}$ starting from the models' steady states.

\section{Results}

\subsection{The hiPSC-CM population}

Out of the 10000 hiPSC-CM models generated by sampling, only 1355 were accepted in the final population after calibration. A global view of the hiPSC-CM population is reported in Figure 1, while the biomarker variability is reported in Table 1. In Figure 2 we show

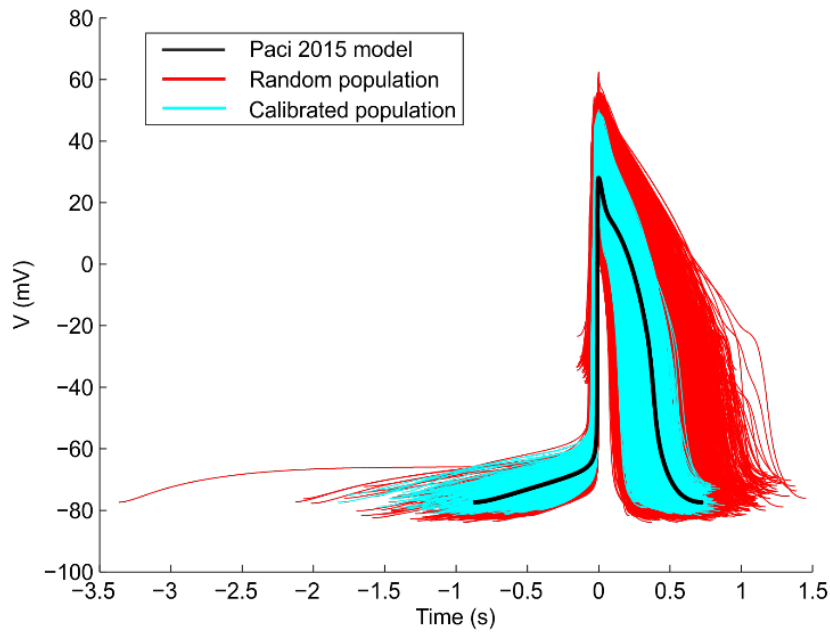

Figure 1. Accepted and rejected APs after calibration. APs upstrokes were aligned and APs were plotted from one MDP to the next one.

Table 1. Biomarkers of the accepted hiPSC-CM models. The lower (LB) and upper (UB) bounds for each biomarker are reported in bold. Only models whose biomarkers are all included in [LB, UB] are accepted in the final population.

\begin{tabular}{lllll}
\hline Biomarker & LB & UB & $\mu$ & $\sigma$ \\
\hline Rate $(\mathrm{bpm})$ & $\mathbf{0}$ & $\mathbf{2 0 9}$ & 54 & 12 \\
MDP $(\mathrm{mV})$ & $\mathbf{- 8 9}$ & $\mathbf{- 4 4}$ & -76 & 2 \\
Peak (mV) & $\mathbf{1 7}$ & $\mathbf{5 8}$ & 35 & 8 \\
APA (mV) & $\mathbf{7 6}$ & $\mathbf{1 3 9}$ & 111 & 9 \\
VMax $(\mathrm{V} / \mathrm{s})$ & $\mathbf{- 2 7}$ & $\mathbf{8 2}$ & 35 & 22 \\
APD $_{10}(\mathrm{~ms})$ & $\mathbf{2 0}$ & $\mathbf{1 2 8}$ & 69 & 26 \\
APD $_{20}(\mathrm{~ms})$ & $\mathbf{0}$ & $\mathbf{2 9 0}$ & 146 & 44 \\
APD $_{30}(\mathrm{~ms})$ & $\mathbf{5 9}$ & $\mathbf{3 0 1}$ & 220 & 53 \\
APD $_{50}(\mathrm{~ms})$ & $\mathbf{0}$ & $\mathbf{6 0 1}$ & 301 & 69 \\
APD $_{70}(\mathrm{~ms})$ & $\mathbf{1 4 6}$ & $\mathbf{6 3 1}$ & 343 & 78 \\
APD $_{90}(\mathrm{~ms})$ & $\mathbf{1}$ & $\mathbf{7 0 5}$ & 384 & 84 \\
\hline
\end{tabular}

how the hiPSC-CMs population covers the biomarker spaces.

\subsection{IKr block}

The $90 \%$ block of $\mathrm{I}_{\mathrm{Kr}}$ on the 1355 hiPSC-CM models, generated three different profiles: 562 models with normal repolarization (REPO), 336 models showing EADs (EAD) and 457 which failed to repolarize (REPOFAIL). Three illustrative APs are reported in Figure 3. For the REPO models, the mean $\triangle \mathrm{APD}_{90}$ prolongation is $(\mu \pm$ SEM) $723 \pm 12 \mathrm{~ms}$. To assess the 

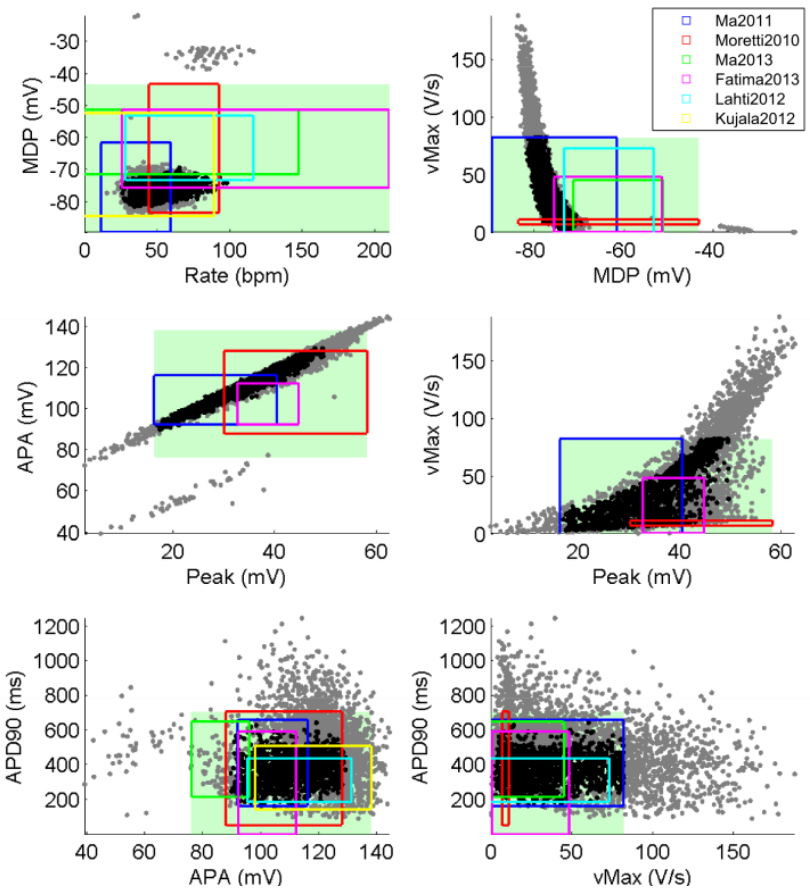

Figure 2. Scatter plots showing biomarkers' values for all the hiPSC-CM models (accepted - black dots; rejected grey dots). Each dataset is here represented as a colored rectangle. The filled green rectangle represents the overall space of the acceptable biomarker values.

mechanisms underlying the occurrence of arrhythmias, we compared the ionic currents among the three classes. Significant differences were identified for $\mathrm{I}_{\mathrm{CaL}}, \mathrm{I}_{\mathrm{Ks}}, \mathrm{I}_{\mathrm{Kr}}$, $\mathrm{I}_{\mathrm{K} 1}, \mathrm{I}_{\mathrm{NaCa}}$ and $\mathrm{I}_{\mathrm{NaK}}$, as shown in Figure 4.

\section{Discussion and conclusions}

In this paper, we presented a new application of the POM paradigm: generating a population of hiPSC-CM in silico models. Our study shows that in a context of extreme variability of the experimental data (Figure 2) a single in silico model, although informative, results partially inadequate to capture the complexity of the electrophysiological behavior of a cellular type. In particular for safety pharmacology purposes, a population of hiPSC-CM models provides a more comprehensive picture on the responses to hiPSC-CMs to drugs. In spite of the fact that a prototypical block was here simulated, the responses of the population models were quite various: from the expected prolongation of APD, to the occurrence of EADs and repolarization failures. We ascribe such different behaviors to a different equilibrium of the ionic currents (in this case their maximum conductances), that in REPO models allow normal APs, but in the EAD and REPOFAIL models, are not able to
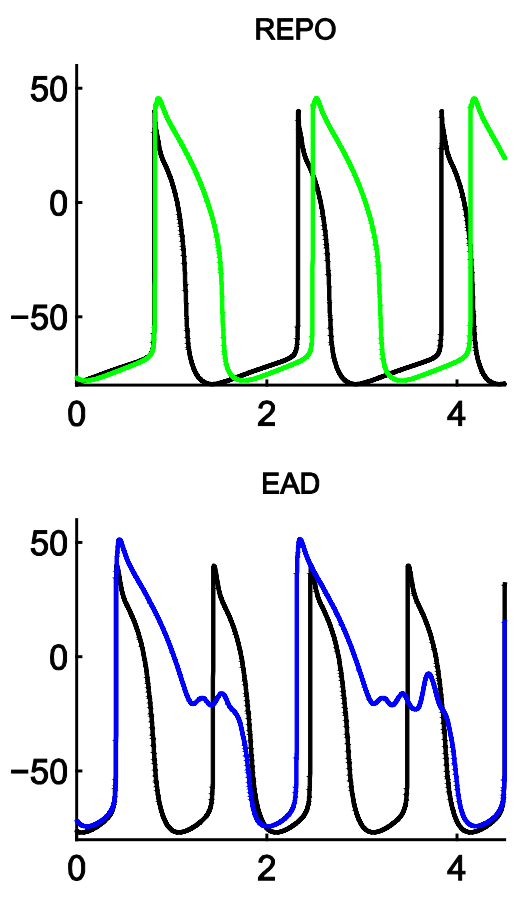

REPOFAIL

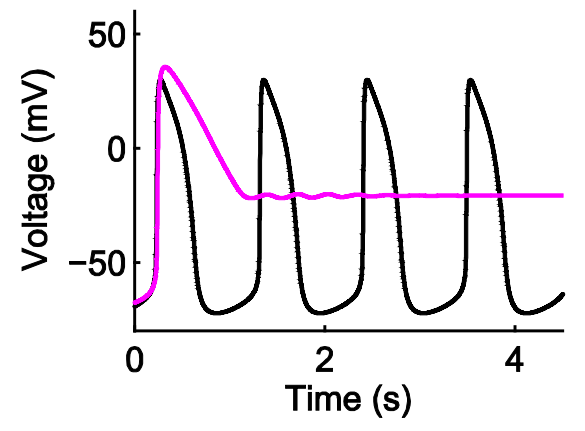

Figure 3. AP comparison before and after $90 \% \mathrm{I}_{\mathrm{Kr}}$ block.

withstand a strong block of $\mathrm{I}_{\mathrm{Kr}}$. The lower $\mathrm{I}_{\mathrm{Ks}}$ and $\mathrm{I}_{\mathrm{K} 1}$ expression level does not surprise. In conditions of almost completely blocked $\mathrm{I}_{\mathrm{Kr}}, \mathrm{I}_{\mathrm{Ks}}$ is expected to compensate the APD prolongation: the REPO models have the greatest $\mathrm{I}_{\mathrm{Ks}}$. In cells like hiPSC-CMs, characterized by a smaller $\mathrm{I}_{\mathrm{K} 1}$ compared to adult cells, an even smaller $\mathrm{I}_{\mathrm{K} 1}$ can hardly stabilize the membrane potential before the next AP. Similar considerations can be made for $\mathrm{I}_{\mathrm{NaK}}$, outward current which is greater in REPO cells, and $\mathrm{I}_{\mathrm{NaCa}}$, which is an inward current during the repolarization thus destabilizing it in EAD and REPOFAIL cells. On the other hand, the stronger $\mathrm{I}_{\mathrm{Kr}}$ in EADs and REPOFAIL, compared to REPO, made us speculate on the fact that in models where $\mathrm{I}_{\mathrm{Kr}}$ is highly expressed, a $90 \%$ block can have more dramatic effects than in cells where $\mathrm{I}_{\mathrm{Kr}}$ is smaller. The significance of $\mathrm{I}_{\mathrm{CaL}}$ in the EAD class can be due to the fact that EADs are generated by reactivation of 

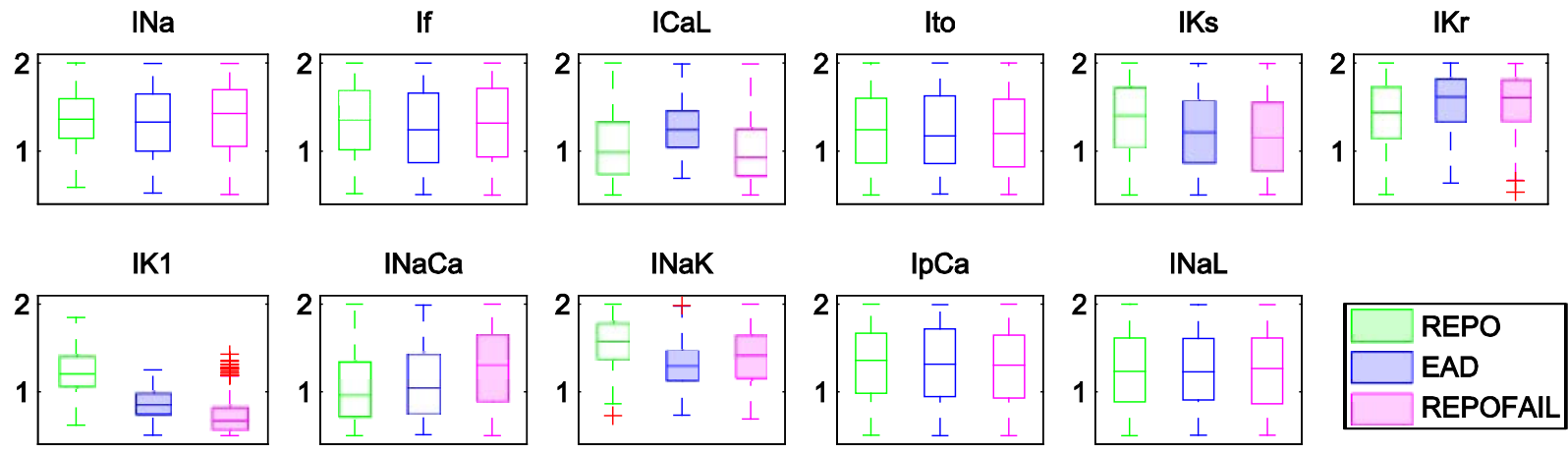

Figure 4. Distribution of the maximum currents for the 11 sampled currents. Filled boxplots represents statistically significant differences $(p$-value $<0.01)$ compared to the REPO class.

$\mathrm{I}_{\mathrm{CaL}}$ upon APD prolongation due to $\mathrm{I}_{\mathrm{Kr}}$ block. The number of repolarization abnormalities depends also by the speeded up $\mathrm{I}_{\mathrm{CaL}}$ recovery from inactivation. In conclusion, POM overcomes the limitations of traditional models and makes in silico modeling even more suitable for safety pharmacology assays. Moreover, we identified the ionic currents affecting the most the development of arrhythomgenic phenomena consequent to a hERG block.

\section{Acknowledgements}

Dr. Michelangelo Paci was supported by the Finnish Cultural Foundation (Central Fund) and the European Society of Cardiology (First Contact Initiative Grant). The authors also thank CSC - IT Centre for Science (Finland) and ARC - Advanced Research Computing of University of Oxford (UK) for computational resources.

\section{References}

[1] Sager PT, Gintant G, et al. Rechanneling the cardiac proarrhythmia safety paradigm: a meeting report from the Cardiac Safety Research Consortium. Am Heart J. Mosby, Inc.; 2014;167(3):292-300.

[2] Paci M, Hyttinen J, et al. Human induced pluripotent stem cell-derived versus adult cardiomyocytes: an in silico electrophysiological study on effects of ionic current block. Br J Pharmacol. 2015;172(21):5147-60.

[3] Paci M, Hyttinen J, et al. Computational models of ventricular- and atrial-like human induced pluripotent stem cell derived cardiomyocytes. Ann Biomed Eng. 2013;41(11):2334-48.
[4] Ma J, Guo L, et al. High purity human-induced pluripotent stem cell-derived cardiomyocytes: electrophysiological properties of action potentials and ionic currents. AJP - Hear Circ Physiol. 2011;301(5):H2006-17.

[5] Britton OJ, Bueno-Orovio A, et al. Experimentally calibrated population of models predicts and explains intersubject variability in cardiac cellular electrophysiology. Proc Natl Acad Sci U S A. 2013;110(23):E2098-105.

[6] Moretti A, Bellin M, et al. Patient-specific induced pluripotent stem-cell models for long-QT syndrome. N Engl J Med. 2010;363(15):1397-409.

[7] Ma D, Wei H, et al. Modeling type 3 long QT syndrome with cardiomyocytes derived from patient-specific induced pluripotent stem cells. Int J Cardiol. Elsevier Ireland Ltd; 2013;168(6):5277-86.

[8] Fatima A, Kaifeng S, et al. The disease-specific phenotype in cardiomyocytes derived from induced pluripotent stem cells of two long QT syndrome type 3 patients. PLoS One. 2013;8(12):e83005.

[9] Lahti AL, Kujala VJ, et al. Model for long QT syndrome type 2 using human iPS cells demonstrates arrhythmogenic characteristics in cell culture. Disease Models \& Mechanisms. 2012. p. 220-30.

[10]Kujala K, Paavola J, et al. Cell model of catecholaminergic polymorphic ventricular tachycardia reveals early and delayed afterdepolarizations. PLoS One. 2012;7(9):e44660.

Address for correspondence.

Michelangelo Paci

Korkeakoulunkatu 3, SM 312, FI-33720 Tampere, Finland michelangelo.paci@tut.fi 